\title{
Regression games
}

\section{Miklós Pintér}

Received: date / Accepted: date

\begin{abstract}
The solution of a $T U$ cooperative game can be a distribution of the value of the grand coalition, i.e. it can be a distribution of the payoff (utility) all the players together achieve. In a regression model, the evaluation of the explanatory variables can be a distribution of the overall fit, i.e. the fit of the model every regressor variable is involved. Furthermore, we can take regression models as $T U$ cooperative games where the explanatory (regressor) variables are the players. In this paper we introduce the class of regression games, characterize it and apply the Shapley value to evaluating the explanatory variables in regression models. In order to support our approach we consider Young (1985)'s axiomatization of the Shapley value, and conclude that the Shapley value is a reasonable tool to evaluate the explanatory variables of regression models.
\end{abstract}

Keywords $T U$ game - Shapley value · Young's axiomatization of the Shapley value $\cdot$ Linear regression model

\section{Introduction}

Consider a regression model where there are one explained and finitely many explanatory (regressor) variables, moreover assume that all the variables are random variables (on a fixed measure space) with finite variance. Then it comes the natural question, how important are the regressor variables in the given regression model?

Financial supports by the Hungarian Scientific Research Fund (OTKA) and the János Bolyai Research Scholarship of the Hungarian Academy of Sciences are gratefully acknowledged.

Miklós Pintér

Corvinus University of Budapest

Department of Mathematics

1093 Hungary, Budapest, Fővám tér 13-15.

E-mail: miklos.pinter@uni-corvinus.hu 
In this paper we apply game theory to giving an answer to the above question. First, we introduce the concept of regression games. A regression game is a transferable utility $(T U)$ cooperative game such that the players are the regressor variables, and it is generated by a regression model in the way the value of a coalition is the difference between the variance (square of the norm) of the explained variable and the square of the distance of the explained variable from the vector space spanned by the given coalition of regressor variables (this the least squares method is about). Our first main result is that we characterize the class of regression games and show that it is a finite convex cone such that the interior of the class of monotone games is its proper subset, and it is a proper subset of the class of monotone games.

The Shapley value (Shapley, 1953) is probably the most popular solution concept for $T U$ cooperative games, henceforth games. We apply the Shapley value to measuring the importance of the regressor variables of regression models. To sum up, we correspond every regression model to a game, and apply a well-known solution concept, the Shapley value, to answering the question of how important the regressor variables are.

There are, however, many other solution concepts for games. In order to give solid reasons for our choice we consider Young (1985)'s axiomatization of the Shapley value. As an application of Pintér (2011)'s result we show that the considered axioms - which are very reasonable ones from the viewpoint of statistics / econometrics, we discuss it in the next paragraph - characterize the Shapley value on the class of regression games. In other words, if one accepts these reasonable statistical requirements (axioms), then she gets that the only evaluation method meeting these axioms (requirements) is the Shapley value.

We impose three axioms, their statistical interpretations are as follows: (1) let the total fit be decomposed among the regressor variables, (2) if two regressor variables are equivalent, i.e. they are indistinguishable in the given regression model, then let they be evaluated equally, (3) if a regressor variable performs better in one regression model than in an other, then let it be evaluated higher in the former model than in the latter. Our second main result is that the set of the above desirability criteria is equivalent with the Shapley value, i.e. accepting these criteria implies the acceptance of the Shapley value, and vice versa.

The main cornerstones of the application of the Shapley value to the discussed statistical / econometric problem are as follows. Chevan and Sutherland (1991) were the first who applied the Shapley value to evaluating explanatory variables. They had distributed the coefficient of determination $\left(R^{2}\right)$ among the explanatory variables in the way the Shapley value does. Chevan and Sutherland (1991) did not recognize that they had used the Shapley value, that was pointed out only later by Stufken (1992) (this is a clear evidence for that the idea of applying the Shapley value to measuring the relative importance of regressor variables had emerged naturally, and independently from game theory).

Lipovetsky and Conklin (2001) also applied the Shapley value to evaluating explanatory variables in regression models. They used this method in order 
to avoid the problem of multicollinearity. Lipovetsky and Conklin (2001) did not know the above papers, hence they did not face the fact that their results were new only to some extent.

It also makes sense to mention that Lipovetsky and Conklin (2001) and Lipovetsky and Conklin (2010) showed that not only the importance of the predictors can be evaluated by the Shapley value of their contribution to the theoretical variance (multiple determination), but also the coefficients of the regression model itself can be re-estimated due to the found Shapley value of the predictors.

It is also worth noticing that the Shapley Value has been successfully applied in statistics not only for linear regression modeling but also in several other statistical decision making problems, see for instance: Grabisch (1996), Powaga and Lipovetsky (2004), Conklin and Lipovetsky (2005), Lipovetsky (2006), Lipovetsky (2007) and Lipovetsky (2008) among others.

The papers Babat (2008) and Feldman (2007) also use game theory to evaluate the regressor variables of regression models, however, Babat (2008)'s approach is completely different from ours (in his model the observations are the players), and Feldman (2007) does not characterize his class of "regression games". As we have already mentioned above, in a regression game the players are the regressor variables, so in a certain sense we consider how these players interact or cooperate. Grabisch and Roubens (1999) consider the interaction or cooperation of players in $T U$ games, so our paper is related to theirs.

Finally, the papers Grömping (2007), Grömping and Landau (2010) and Lipovetsky and Conklin (2010) clearly show that the problem we discuss in this paper, is in the very focus of recent statistical / econometric research.

The paper is organized as follows: in Section 2 we define and characterize the class of regression games, and in Section 3 we present our axiomatization result.

\section{Regression games}

Notation: $|N|$ and $\mathcal{P}(N)$ denote respectively the cardinality and all subsets of set $N . A \subset B$, if $A \subseteq B$ and $A \neq B$. Cone $(S)$ denotes the smallest convex cone containing set $S$, furthermore, $\operatorname{Lin}\left(\left\{\xi_{1}, \ldots, \xi_{k}\right\}\right)$ denotes the linear subspace spanned by vectors $\xi_{1}, \ldots, \xi_{k}$. Let $A$ be a set in a topological space, then $\operatorname{Int}(A)$ denotes the interior of set $A$. Let $X$ be an inner product space and $A \subseteq X$ be a subspace, then $A^{\perp}$ denotes the orthogonal complement of set $A$. Moreover, $A \subseteq X$ is a cone, if $\forall \alpha>0: \alpha A \subseteq A$, where $X$ is a vector space (over the real field).

Let $N$ be a non-empty finite set. Then $v: \mathcal{P}(N) \rightarrow \mathbb{R}$ such that $v(\emptyset)=0$ is called $T U$ (transferable utility) cooperative game with player set $N$, henceforth game. $\mathcal{G}^{N}$ denotes the class of games with player set $N$. It is well known that $\mathcal{G}^{N}$ and $\mathbb{R}^{2^{|N|}-1}$ are isomorphic (as vector spaces), therefore w.l.o.g. we can assume that $N=\{1, \ldots,|N|\}$, moreover, in this paper we use the following order on $\mathcal{P}(N): \forall A, B \in \mathcal{P}(N) A$ is "before" $B$, if $|A|<|B|$, or $|A|=|B|$ and 
$A$ is before $B$ in the lexicographic order. E.g. if $N=\{1,2,3\}$, then the fixed order is $\{\{1\},\{2\},\{3\},\{1,2\},\{1,3\},\{2,3\},\{1,2,3\}\}$.

It is also important to note that at certain points in our discussion we refer to $\mathcal{G}^{N}$ as a normed vector space. Since on a finite dimensional vector space all norms are topologically equivalent, and we use only the concept of convergence, we do not need to specify the norm on $\mathcal{G}^{N}$.

Game $v \in \mathcal{G}^{N}$ is monotone, if $\forall S, T \subseteq N$ such that $S \subseteq T: v(S) \leq v(T)$; it is superadditive, if $\forall S, T \subseteq N$ such that $S \cap T=\emptyset: v(S)+v(T) \leq v(S \cup T)$; it is subadditive, if $\forall S, T \subseteq N$ such that $S \cap T=\emptyset: v(S)+v(T) \geq v(S \cup T)$; it is essential, if $v(N)>\sum_{i \in N} v(\{i\})$.

The dual of game $v \in \mathcal{G}^{N}$ is a game $\bar{v} \in \mathcal{G}^{N}$ such that $\forall S \subseteq N: \bar{v}(S)=$ $v(N)-v(N \backslash S)$. Let $T \subseteq N \backslash \emptyset$ be an arbitrary set, then

$$
u_{T}(S)= \begin{cases}1, & \text { if } T \subseteq S \\ 0 & \text { otherwise }\end{cases}
$$

is called unanimity game on coalition $T$.

In this section we consider linear regression models (henceforth regression models). It is our goal to establish theoretically the application of the Shapley value to evaluating explanatory variables in regression models.

Let $\eta$ be the explained and $\xi_{i}, i \in N,|N|<\infty$ be the explanatory variables of a regression model, henceforth $\left(\eta,\left\{\xi_{i}\right\}_{i \in N}\right)$. We examine the problem in an abstract way, i.e. we do not care about estimations and other empirical concepts, we use (known) random variables with finite variance.

Definition 1 Let $N$ be the player set, in other words the players are the explanatory variables.

See the following problem $(S \subseteq N$ is an arbitrary set):

$$
\begin{aligned}
& \operatorname{var}(\eta)-\operatorname{var}\left(\eta-\sum_{i \in S} \beta_{i} \xi_{i}\right) \rightarrow \max \\
& \beta_{i} \in \mathbb{R}, i \in S
\end{aligned}
$$

Notice that in the objective function of (1) the second term is the square of the distance of the explained variable from the linear space spanned by the regressor variables of $S$. Since the second term has a negative sign and we maximize the objective function, the optimal solution of (1) is the least square solution.

Because of that the value of the empty coalition be zero, we compare this square of distance to the square of the distance of the explained variable from the origin (0), i.e. to the square of the norm of the explained variable. Put it differently, in (1) we apply the Least Squares Method to the regression problem with regressor variables of $S$, and take the difference of a constant (the variance of the explained variable) and the least square.

Definition 2 Let $\left(\eta,\left\{\xi_{i}\right\}_{i \in N}\right)$ be a regression model. $\forall S \in \mathcal{P}(N)$ let $v(S)$ be the optimal solution of (1). 
The value of a coalition $(S)$ is the optimal solution of model (1), it is similar to $S S R$ (regression sum of squares). Chevan and Sutherland (1991) and Lipovetsky and Conklin (2001) used the coefficient of determination $\left(R^{2}\right)$, hence we differ from them at this point.

Corollary 1 v is a (TU cooperative) game.

Proof It is the direct corollary of Definitions 1 and 2

We can regard $v$ as a set of linear regression problems determined by the given regression model.

Definition 3 Let $\left(\eta,\left\{\xi_{i}\right\}_{i \in N}\right)$ be a regression model, $v \in \mathcal{G}^{N}$ be the generated game and $\Pi$ be a partition of $N$. Then the restricted regression model of $\left(\eta,\left\{\xi_{i}\right\}_{i \in N}\right)$ on $\Pi$ denoted by $\left(\eta,\left\{\xi_{i}\right\}_{i \in N}, \Pi\right)$ is the model where the players are the elements of $\Pi$. Moreover, $v^{\Pi} \in \mathcal{G}^{\Pi}$ denotes the restricted game, i.e. it denotes the game generated by $\left(\eta,\left\{\xi_{i}\right\}_{i \in N}, \Pi\right)$.

Put the above definition differently, in a restricted game some players are concentrated to form super players, and these new players, the super players, are the players in the restricted game. The next example illustrates the concept of Definition 3

Example 1 Let the covariance matrix of a regression model (there are three regressor variables in this model) be

$$
\left(\begin{array}{rrrr}
1 & 0 & 1 & 1 \\
0 & 1 & -1 & 0 \\
1 & -1 & 4 & 2 \\
1 & 0 & 2 & 3
\end{array}\right),
$$

where the elements in the diagonal are the variances of the random variables $((1,1)$ is the variance of the explained variable), and the other elements are the covariances.

The generated game is as follows: $v=\left(0, \frac{1}{4}, \frac{1}{3}, \frac{1}{3}, \frac{1}{3}, \frac{3}{8}, \frac{2}{5}\right)$. It is important to notice that, the regression game $v$ is monotone, but it is not super-, or subadditive or essential.

Furthermore, let $\Pi=\{\{1,3\},\{2\}\}$ be a partition of the player set. Then $v^{\Pi}=\left(\frac{1}{3}, \frac{1}{4}, \frac{2}{5}\right)$.

The intuition behind Definition 3 is the following: in some cases the modeler (statistician) is interested in the evaluation of not only a single regressor variable, but rather a group of them. Put it differently, the modeler concentrates some explanatory variables into groups, tackle them as players (super players) and measure the explaining power of these super players.

For the reasonableness of super players (variables), consider the following example. In a data set we have a nominal variable with three "values", and 
we want to put this nominal variable into a regression model. Then we have to convert this nominal variable into two dummy variables, in the way every dummy variable belongs to a "value" of the nominal variable. Furthermore, if we want to measure the importance of the original nominal variable in the regression model, then we have to take the three generated dummy variables as a super variable (player), and put all or none of them into the model.

Definition 4 Let $N$ be a non-empty finite set. $v \in \mathcal{G}^{N}$ is a regression game if $\exists M$ finite set of regressor variables, $\exists \eta$ explained variable and $\exists \Pi$ partition of $M$ such that $v$ is generated by $(\eta, M, \Pi)$ (notice that $|\Pi|=|N|$ ). Furthermore, let $\mathcal{G}_{R}^{N}$ denote the class of regression games with player set $N$.

In other words, a regression game with player set $N$ is a game such that it is a restricted game of a game which is generated by a regression model in the way (1) defines it.

The next proposition shows the power of the Definition 4 . Henceforth, when we write explanatory (regressor) variable then we mean a super variable (a set of variables that can be singleton).

Proposition $1 \mathcal{G}_{R}^{N}$ is a convex cone.

We split the proof into two lemmata.

Lemma $1 \mathcal{G}_{R}^{N}$ is a cone.

Proof If we multiply the explained variable in (1) by $\alpha>0$, then the generated game will be the $\alpha^{2}$ multiplication of the original game. Put it differently, if we multiply the covariance matrix of the given model by $\alpha>0$, then the generated game will be the $\alpha$ multiplication of the original game.

Lemma $2 \mathcal{G}_{R}^{N}$ is a convex set.

The strategy of the proof is the following. Pick two arbitrary regression games and their convex weights, take the two regression models which generate the two games in the way such that any random variable of the first is independent from any of the second (in the proof they are only orthogonal), then put the (convex) weighted modifications of them together. Finally, a reduced game of the game generated by this regression model is the convex combination of the two original games.

Proof Let $w, z \in \mathcal{G}_{R}^{N}$ and $\alpha \in[0,1]$. Then $\exists M^{w}=\left\{\xi_{1}^{w}, \ldots, \xi_{m}^{w}\right\}, M^{z}=$ $\left\{\xi_{1}^{z}, \ldots, \xi_{m^{z}}^{z}\right\}$ sets of regressor variables, $\exists \eta^{w}, \eta^{z}$ explained variables, $\exists \Pi^{w}, \Pi^{z}$ partitions of $M^{w}$ and $M^{z}$ respectively such that $w$ and $z$ are generated by $\left(\eta^{w}, M^{w}, \Pi^{w}\right),\left(\eta^{z}, M^{z}, \Pi^{z}\right)$ respectively and $\operatorname{Lin}\left(\left\{\eta^{w}\right\} \cup M^{w}\right) \subseteq \operatorname{Lin}^{\perp}\left(\left\{\eta^{z}\right\}\right.$ $\left.\cup M^{z}\right)$.

Lemma 1 implies that $\alpha w \in \mathcal{G}_{R}^{M^{w}},(1-\alpha) z \in \mathcal{G}_{R}^{M^{z}}$ and $\alpha w$ is generated by $\left(\sqrt{\alpha} \eta^{w}, M^{w}\right)$ and so is $(1-\alpha) z^{\prime}$ by $\left(\sqrt{(1-\alpha)} \eta^{z}, M^{z}\right)$.

Consider the model in which the explained variable is $\sqrt{\alpha} \eta^{w}+\sqrt{(1-\alpha)} \eta^{z}$ and the explanatory variables are $M^{w} \cup M^{z}$. Moreover, let $\Pi$ be a partition 
of $M^{w} \cup M^{z}$ such that it consists of the sets $V_{i}=W_{i} \cup Z_{i}$, where $V_{i}$ is the $i$ th element of partition $\Pi$, so are $W_{i}$ and $Z_{i}$ of partitions $\Pi^{w}$ and $\Pi^{z}$ respectively, $i=1, \ldots,|N|$, hence $|\Pi|=|N|$.

Let $v$ be generated by $\left(\sqrt{\alpha} \eta^{w}+\sqrt{(1-\alpha)} \eta^{z}, M^{w} \cup M^{z}, \Pi\right)$. Then $v \in \mathcal{G}_{R}^{N}$, and it is an easy calculation (remember $\left.\operatorname{Lin}\left(\left\{\eta^{w}\right\} \cup M^{w}\right) \subseteq \operatorname{Lin}^{\perp}\left(\left\{\eta^{z}\right\} \cup M^{z}\right)\right)$ to see that $v=\alpha w+(1-\alpha) z . w, z$ and $\alpha$ were arbitrarily fixed, hence the proof is complete.

Proof (The proof of Proposition 1) It is the direct corollary of Lemmata 1 and 2.

Next we show that $\mathcal{G}_{R}^{N}$ is a proper subset of the class of monotone games.

Lemma 3 If $|N| \geq 2$, then $\mathcal{G}_{R}^{N} \subset \operatorname{Mon}^{N}$, where $\operatorname{Mon}^{N}$ denotes the class of monotone games with player set $N$.

Proof The proof of that every regression game is monotone is left for the reader. However, if $|T|>1$, then the unanimity game $u_{T}$ is monotone and $u_{T} \notin \mathcal{G}_{R}^{N}$. If $\forall i \in T: \xi_{i} \in \operatorname{Lin}^{\perp}(\{\eta\})$, in other words $v\left(\left\{\xi_{i}\right\}\right)=0$, then $\forall \xi^{*} \in$ $\operatorname{Lin}\left(\left\{\xi_{i}\right\}_{i \in T}\right): \xi^{*} \in \operatorname{Lin}^{\perp}(\{\eta\})$, put it differently $v(T)=0$.

Naturally, in the trivial case of $|N|=1 \mathcal{G}_{R}^{N}$ and the class of monotone games coincide.

Proposition $2 \operatorname{Int}\left(\operatorname{Mon}^{N}\right) \subset \mathcal{G}_{R}^{N}$.

To prove the above proposition we need the following notion.

Definition 5 Let $T \subseteq \mathcal{P}(N)$ be such that $T \backslash\{\emptyset\} \neq \emptyset$ and $\forall A, B \in T$ : $|A|=|B|$. Furthermore, let

$$
g_{T}(S)=\left\{\begin{array}{ll}
1, & \text { if } \exists A \in T \text { such that } A \subseteq S \\
0 & \text { otherwise }
\end{array} .\right.
$$

Then $g_{T}$ is called generalized unanimity game on coalitions of $T$.

It is clear that any unanimity game and the dual of any unanimity game are generalized unanimity games (that is a reason for the naming). E.g. $\bar{u}_{N}=$ $g_{\{A \subseteq N|| A \mid=1\}}$ and $u_{N}=g_{\{N\}}$. However, e.g. the generalized unanimity game $g_{\{A, B\}}, A \neq B$ is neither a unanimity game nor the dual of any of them.

An other reason for the name of $g_{T}$ is the following: $\forall S \subseteq N: g_{T}(S)=$ $\max _{A \in T} u_{A}(S)$. In other words, $g_{T}$ is the upper envelope of unanimity games $\left\{u_{A}\right\}_{A \in T}$

Next we characterize the class of monotone games.

Lemma 4 The class of monotone games (for any player set) is a finite convex cone spanned by the generalized unanimity games. 
Proof Let $v \in \mathcal{G}^{N}$ be a monotone game. Consider the following algorithm:

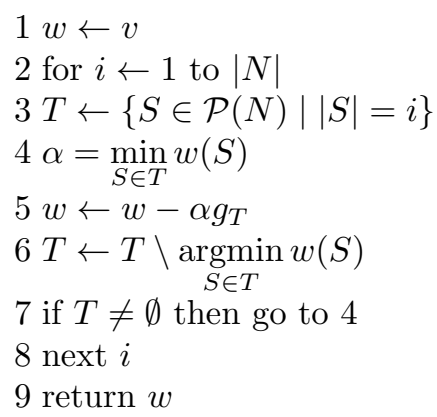

It is an easy calculation to show that the above algorithm returns with 0 , moreover, $\alpha$ never hits the negative region, hence

$$
v \in \text { Cone }\left(\left\{g_{T}\right\}_{T \subseteq \mathcal{P}(N), T \backslash\{\emptyset\} \neq \emptyset, \forall A, B \in T:|A|=|B|}\right) .
$$

$v$ was an arbitrary monotone game, therefore, any monotone game is in the convex cone spanned by the generalized unanimity games.

The following lemmata show that the class of regression games contains the interior of the class of monotone games.

Lemma 5 Let $u_{T} \in \mathcal{G}^{N}$ be a unanimity game. Then $\bar{u}_{T} \in \mathcal{G}_{R}^{N}$.

Proof Consider the following regression model:

$$
\begin{aligned}
& \operatorname{var}(\eta) \quad=1 \\
& \operatorname{cov}\left(\xi_{i}, \eta\right)= \begin{cases}1, & \text { if } i \in T \\
0 & \text { otherwise }\end{cases} \\
& \operatorname{cov}\left(\xi_{i}, \xi_{j}\right)= \begin{cases}1, & \text { if } i, j \in T \\
0 & \text { otherwise }\end{cases}
\end{aligned}
$$

Then the regression game generated by the above regression model is $\bar{u}_{T}$.

Lemma 6 Let $N$ be a non-empty finite set and $T \subseteq \mathcal{P}(N)$ be such that $T \backslash$ $\{\emptyset\} \neq \emptyset$ and $\forall A, B \in T:|A|=|B|$. Then $\exists A_{i} \subseteq \mathbb{N}, A_{i} \neq \emptyset, i=1, \ldots,|N|$, and $\exists k \in \mathbb{N}$ such that $\forall S \subseteq N: g_{T}(S)=1$ if and only if $\left|\bigcup_{i \in S} A_{i}\right| \geq k$.

Proof Let $T \subseteq \mathcal{P}(N)$ be such that $T \backslash\{\emptyset\} \neq \emptyset$ and $\forall A, B \in T:|A|=|B|$. The following algorithm produces the sets $A_{i}$ and $k$. 
$1 \quad \alpha \leftarrow|A|, A \in T$

2 for $i \leftarrow 1$ to $|N|$

$3 A_{i} \leftarrow\{i\}$

4 next $i$

$5 \quad k \leftarrow \alpha$

\# At this stage $\forall S \in \mathcal{P}(N):\left|\bigcup_{i \in S} A_{i}\right| \geq k$, if and only if $|S| \geq \alpha$.

\# Next we manipulate sets $S$ such that $S \in \mathcal{P}(N),|S| \geq \alpha$

$\#$ and $g_{T}(S)=0$.

$6 j \leftarrow|N|+1$

7 for $S \in \mathcal{P}(N),|S| \geq \alpha, g_{T}(S)=0$

8 if $\left|\bigcup_{i \in S} A_{i}\right|<k$ then goto 13

$9 \quad A_{i} \leftarrow A_{i} \cup\{j\} i \notin S$

$10 j \leftarrow j+1$

$11 k \leftarrow k+1$

11 go to 8

13 next $S$

\# It is clear that, if $Z \subseteq N, Z \backslash S \neq \emptyset$, then $\left|\bigcup_{i \in Z} A_{i}\right|$ increases by one,

\# and so does $k$. In the other case $(Z \subseteq S) g_{T}(Z)=0$, hence it is OK.

\# that $\left|\bigcup_{i \in Z} A_{i}\right|$ does not increase and $k$ does.

14 return $A_{i}, i \in N, k$

It is easy to see that for the returned sets $A_{i}$ and $k, g_{T}(S)=1$, if and only if $\left|\bigcup_{i \in S} A_{i}\right| \geq k$.

It is worth noticing that in line 7 we do not specify the order in which the algorithm applies the given sets, that order can be arbitrary.

Lemma 7 Let $T \subseteq \mathcal{P}(N)$ be such that $T \backslash\{\emptyset\} \neq \emptyset$ and $\forall A, B \in T:|A|=|B|$. Then $\forall \varepsilon>0: \exists v \in \mathcal{G}_{R}^{N}$ such that $\left\|v-g_{T}\right\|<\varepsilon$.

Proof Since the considered space of the random variables with finite variance is an infinite dimensional Hilbert space, we can work in $\mathbb{R}^{n}$ (with the standard inner product), where $n$ is a natural number.

Let $\varepsilon>0$. First we show that $\forall n \in\{1, \ldots,|N|\}, \forall T=\{A \subseteq N|| A \mid=n\}$ : $\exists v \in \mathcal{G}_{R}^{N}$ such that $\left\|v-g_{T}\right\|<\varepsilon$.

For the case of $n=1$, (when $\left.g_{T}=\bar{u}_{N}\right)$ see Lemma 5

$n=2$ : Let $\eta=(0,1)$ and $\alpha>0$. Furthermore, let $\xi_{i}^{\alpha}=\left(\frac{i}{\alpha}, 1\right), i=$ $1, \ldots,|N|$, and $v_{T}^{\alpha}$ be generated by $\left(\eta,\left\{\xi_{i}^{\alpha}\right\}_{i=1}^{|N|}\right)$. Then it is easy to see that $g_{T}=\lim _{\alpha \rightarrow 0} v_{T}^{\alpha}$. 
$n>2$ : Let $\eta=\frac{1}{\sqrt{n}} \underline{1}$, where $\underline{1} \in \mathbb{R}^{n}$ is the vector with components 1 , and $S(\eta)=\left\{x \in \mathbb{R}^{n} \mid\|x-\eta\|=1\right\}$ (the unit sphere of center $\eta$ ). Furthermore, let $X$ be an $n-1$ dimensional subspace of $\mathbb{R}^{n}$ such that $\eta \in X^{\perp}$, and $\xi_{i}$ be vectors such that $\xi_{i} \in S(\eta) \cap(X+\eta), i=1, \ldots,|N|$ and any $n$ member subset of vectors $\xi_{1}, \ldots, \xi_{|N|}$ is a basis of $\mathbb{R}^{n}$, however, the vector space spanned by any $n-1$ member subset of vectors $\xi_{1}, \ldots, \xi_{|N|}$ does not contain $\eta$ (it is clear that there are such vectors). Moreover, let $p r: \mathbb{R}^{n} \rightarrow X$ be the projection into the subspace $X, \alpha>0$, and $v_{T}^{\alpha}$ be the game generated by $\left(\eta,\left\{\alpha \xi_{i}+(1-\alpha) \operatorname{pr}\left(\xi_{i}\right)\right\}_{i=1}^{|N|}\right)$. Then it is easy to see that $g_{T}=\lim _{\alpha \rightarrow 0} v_{T}^{\alpha}$ (as $\alpha \rightarrow 0 \alpha \xi_{i}+(1-\alpha) \operatorname{pr}\left(\xi_{i}\right)$ is getting closer to $\left.X\right)$.

Next we show that $\forall T \subseteq \mathcal{P}(N)$ such that $T \backslash\{\emptyset\} \neq \emptyset$ and $\forall A, B \in T$, $|A|=|B|: \exists v \in \mathcal{G}_{R}^{N}$ such that $\left\|v-g_{T}\right\|<\varepsilon$. Let $n=|A|, A \in T$. Consider game $w_{T}^{\alpha}$ from the previous paragraph (i.e. $w_{T}^{\alpha}=v_{T}^{\alpha}$ ) where the player set is $\bigcup_{i} A_{i}$ and $n=k$, and the sets $A_{i}$ and $k$ are from Lemma 6. Then we have a regression model in which any $n(k)$ of the regressor variables can fully explain the explained variable $\eta$, but any less than $n(k)$ of them can explain only nothing (asymptotically). Let $\sum_{i} A_{i}$, the direct sum of sets $A_{i}$, be a new player set and the explained variable be retained. Then $\Pi=\left\{A_{1}, \ldots, A_{|N|}\right\}$ is a partition of $\sum_{i} A_{i}$, moreover let $v_{T}^{\alpha}=\left(w_{T}^{\alpha}\right)^{\Pi}$. Then the previous paragraph implies that $g_{T}=\lim _{\alpha \rightarrow 0} v_{T}^{\alpha}$.

The proof of the above lemma shows that the concept introduced in Definition 3 ensures not only that $\mathcal{G}_{R}^{N}$ is a convex cone, but more.

Proof (The proof of Proposition 2) Let $\left\{\alpha_{n}\right\}_{n}$ be a sequence such that $\alpha_{n} \searrow 0$. Then from Proposition 1 and Lemmata 4,7

$$
\operatorname{Int}\left(\operatorname{Mon}^{N}\right) \subseteq \bigcup_{n} \text { Cone }\left\{v_{T}^{\alpha_{n}}\right\}_{T \subseteq \mathcal{P}(N), T \backslash\{\emptyset\} \neq \emptyset, \forall A, B \in T:|A|=|B| \subseteq \mathcal{G}_{R}^{N}},
$$

where $v_{T}^{\alpha_{n}}$ are the regression games constructed in Lemma 7

Furthermore, from Lemma $5 . \mathcal{G}_{R}^{N} \nsubseteq \operatorname{Int}\left(\mathrm{Mon}^{N}\right)$

We can say only two more things:

Lemma 8 If the explanatory variables are uncorrelated (orthogonal), then the generated regression game is additive. Moreover, if two explanatory variables are linearly correlated, then exchanging them has no any impact on the generated regression game.

It is important to see that, the above arguments (Lemma 8) cannot be reversed. If a regression game is additive, then it is not necessary that the explanatory variables are uncorrelated, and if exchanging two regressor variables does not matter, then it does not imply that those are linearly correlated.

Summing up the characterization of $\mathcal{G}_{R}^{N}$, we get the following corollary. 
Corollary 2 The class of regression games $\mathcal{G}_{R}^{N}$ is

1. a convex cone,

2. $\operatorname{Int}\left(\operatorname{Mon}^{N}\right) \subset \mathcal{G}_{R}^{N} \subset \operatorname{Mon}^{N}$,

3. neither open nor closed,

4. not a subset of the class of super-, or subadditive or essential games.

Proof See Example 1, Lemma 3 and Propositions 1, 2,

\section{The Shapley value}

In this section we consider Young (1985)'s axiomatization of the Shapley value on the class of regression games. First we introduce some notions and Young (1985)'s result.

For any $v \in \mathcal{G}^{N}, i \in N$ let $v_{i}^{\prime}(S)=v(S \cup\{i\})-v(S)$, where $S \subseteq N$. Then $v_{i}^{\prime}(S)$ is the marginal contribution of player $i$ to coalition $S$ in game $v$.

Definition 6 Let $v \in \mathcal{G}^{N}$ be a game. Players $i, j \in N$ are equivalent, $i \sim^{v} j$, if $\forall S \subseteq N$ such that $i, j \notin S: v_{i}^{\prime}(S)=v_{j}^{\prime}(S)$. $S$ is an equivalence set in $v$, if $\forall i, j \in S: i \sim^{v} j$.

A solution on set $A \subseteq \mathcal{G}^{N}$ is a function $\psi: A \rightarrow \mathbb{R}^{N}$. In this paper we assume that a solution is a singleton set valued mapping.

Definition 7 Let $v \in \mathcal{G}^{N}$ be a game and

$$
p_{S h}^{i}(S)=\left\{\begin{array}{ll}
\frac{|S| !(|N|-|S|-1) !}{|N| !}, & \text { if } i \notin S \\
0 & \text { otherwise }
\end{array} .\right.
$$

Then $\phi_{i}(v)$, the Shapley value (Shapley, 1953) of player $i$ in game $v$, is the $p_{S h}^{i}$ expected value of $v_{i}^{\prime}$. Put it differently

$$
\phi_{i}(v)=\sum_{S \subseteq N} v_{i}^{\prime}(S) p_{S h}^{i}(S) .
$$

Furthermore, let $\phi$ denote the Shapley solution.

Definition 7 emphasizes the expected value interpretation of the Shapley value.

Definition $8 \psi$, a solution on $A \subseteq \mathcal{G}^{N}$, is / satisfies the

- Pareto optimal $(P O)$, if $\forall v \in A: \sum_{i \in N} \psi_{i}(v)=v(N)$,

- equal treatment property $(E T P)$, if $\forall v \in A:\left(i \sim^{v} j\right) \Rightarrow\left(\psi_{i}(v)=\psi_{j}(v)\right)$,

- strongly monotone $(S M)$, if $\forall v, w \in A:\left(v_{i}^{\prime} \leq w_{i}^{\prime}, i \in N\right) \Rightarrow\left(\psi_{i}(v) \leq\right.$ $\left.\psi_{i}(w)\right)$. 
In the following paragraphs we interpret the above defined properties (axioms).

Grömping (2007) argues that the proper decomposition (the total fit is decomposed among the regressor variables) is an indispensable requirement for an evaluation method. The statistical concept of proper decomposition is equivalent with the game theoretical concept of $P O$.

$E T P$ requires that, if two regressor variables have the same effects on the given regression model, then they be evaluated equally. This axiom can be corresponded to the following property: if exchanging two explanatory variables has no influence on the generated game, then their evaluations be the same.

Property $S M$ is about that, if one compares two models in which the same regressor variable (indexed by the same natural number) performs better (is more useful) in the first model than in the second, then its evaluation be higher in the first model than in the second. We can interpret this requirement as the evaluation should depend on only the performance, it should not biased by other factors.

In our opinion, all the three discussed axioms are reasonable and acceptable from the viewpoint of statistics / econometrics.

Next we focus on Young (1985)'s axiomatization of the Shapley on the class of regression games. First we provide Young (1985)'s results:

Theorem 1 (Young (1985)) $\psi$, a solution on $\mathcal{G}^{N}$, is PO, ETP and $S M$ if and only if $\psi=\phi$, i.e. if and only if it is the Shapley solution.

The next result is our characterization result.

Theorem $2 \psi$, a solution on the class of regression games, is PO, ETP and $S M$ if and only if $\psi=\phi$, i.e. if and only if it is the Shapley solution.

To prove the above theorem we need the following concept.

Definition 9 Set $A \subseteq \mathcal{G}^{N}$ is $M$-closed, if $\forall v \in A$ such that $S \subseteq N$ is an equivalence set in $v$, and $\forall k \in N \backslash S: \exists w \in A$ such that $S \cup\{k\}$ is an equivalence set in $w$ and $w_{k}^{\prime}=v_{k}^{\prime}$.

Pintér (2011) mentions that $\operatorname{Mon}^{N}$ is an $M$-closed class of games. The next proposition is a simplified version of Pintér (2011)'s result.

Proposition 3 Let $A \subseteq \mathcal{G}^{N}$ be an $M$-closed class of games. $\psi$, a solution on set $A$, is PO, ETP and $S M$ if and only if $\psi=\phi$, i.e. if and only if it is the Shapley solution.

Proof See Theorem 3.3 in Pintér 2011).

In order to prove Theorem 2 we need the following lemma.

Lemma 9 Let $v \in \mathcal{G}^{N}$ be a game and

$$
e=\sum_{T} g_{T}
$$

where $T \subseteq \mathcal{P}(N)$ is such that $T \backslash\{\emptyset\} \neq \emptyset$ and $\forall A, B \in T:|A|=|B|$. Then $\forall \varepsilon \in \mathbb{R}, i, j \in N: i \sim^{v} j$ if and only if $i \sim^{v+\varepsilon e} j$. 
Proof It is clear that $\forall i \in N:(v+\varepsilon e)_{i}^{\prime}=v_{i}^{\prime}+\varepsilon e_{i}^{\prime}$. Moreover, it is a slight calculation to see that $\forall i, j \in N: i \sim^{e} j$, therefore the proof is complete.

Proof (The proof of Theorem 2) First we show that $\operatorname{Int}\left(\mathrm{Mon}^{N}\right)$ is an $M$-closed class of games. Let $v \in \operatorname{Int}\left(\mathrm{Mon}^{N}\right)$ be such that $S$ is an equivalence set in $v$, and $k \in N \backslash S$ be a player. Lemma 4 implies that $\exists \varepsilon>0, \exists w \in \operatorname{Mon}^{N}$ such that $v=w+\varepsilon e$, and from Lemma 9 is an equivalence set in $w$. Since $\operatorname{Mon}^{N}$ is an $M$-closed set of games (see Pintér (2011)), $\exists z \in \operatorname{Mon}^{N}$ such that $S \cup\{k\}$ is an equivalence set in $z$ and $z_{k}^{\prime}=w_{k}^{\prime}$. Then from Lemma $9 \cup\{k\}$ is an equivalence set in $z+\varepsilon e \in \operatorname{Int}\left(\operatorname{Mon}^{N}\right)$ and $(z+\varepsilon e)_{k}^{\prime}=v_{k}^{\prime}$, i.e. $\operatorname{Int}\left(\operatorname{Mon}^{N}\right)$ is $M$-closed

Let $v \in \mathcal{G}_{R}^{N}$ and $\varepsilon>0$. Then $v+\varepsilon e \in \operatorname{Int}\left(\mathrm{Mon}^{N}\right)$, from Proposition 2 $v+\varepsilon e \in \mathcal{G}_{R}^{N}$, and from Proposition 3 and the previous paragraph $\psi(v+\varepsilon e)=$ $\phi(v+\varepsilon e)$. Moreover, $S M$ ensures that $\psi(v) \leq \psi(v+\varepsilon e)=\phi(v+\varepsilon e)$. Therefore that $\varepsilon>0$ was arbitrarily chosen, that $\phi$ is continuous and axiom $P O$ imply that $\psi(v)=\phi(v)$.

It is important to see that neither Young (1985)'s nor Moulin (1988)'s proofs do not work in this case, hence the above result is a real application of Pintér (2011)'s alternative proof for Young's axiomatization.

Summing up our results, Theorem 2 implies that on the class of regression games the Shapley value is equivalent with the three reasonable requirements (axioms) discussed in Definition 8. In other words, if we accept the three axioms as basic properties of an evaluation method, then we get the Shapley value since this is the only solution which meets the pre-defined criteria.

It has been the motivation of this paper to establish a solid mathematical background for using the Shapley value as a regressor variable evaluation method in linear regression models. We conclude that applying the Shapley value to evaluating explanatory variables is mathematically and theoretically well founded.

Acknowledgements I thank Ferenc Forgó for calling my attention to the subject of this paper, i.e. to applying the Shapley value to evaluating explanatory variables in regression models. I also thank Ferenc Forgó, Ágota Orosz,Tamás Solymosi, the anonymous referees and the associate editor for their suggestions and remarks. Naturally, all errors are mine.

\section{References}

Babat R (2008) On some classes of balanced games. Mathematical Programming And Game Theory For Decision Making, vol.1. Statistical Science and Interdisciplinary Research, John Wiley \& Sons, New York

Chevan A, Sutherland M (1991) Hierarchical partitioning. The American Statistician 45:90-96

Conklin W, Lipovetsky S (2005) Marketing decision analysis by turf and shapley value. Information Technology and Decision Making 4:5-19 
Feldman B (2007) A theory of attribution. Munich RePEc Personal Archive, ID: 3349

Grabisch M (1996) The representation of importance and interaction of features by fuzzy measures. Pattern Recognition Letters 17:567-575

Grabisch M, Roubens M (1999) An axiomatic approach to the concept of interaction among players in cooperative games. International Journal of Game Theory 28:547-565

Grömping U (2007) Estimators of relative importance in linear regression based on variance decomposition. The American Statistician 61:139-146

Grömping U, Landau S (2010) Do not adjust coefficients in shapley value regression. Applied Stochastic Models in Business and Industry 26:194-202

Lipovetsky S (2006) Entropy criterion in logistic regression and shapley value of predictors. Journal of Modern Applied Statistical Methods 5:121-132

Lipovetsky S (2007) Iteratively re-weighted random-coefficient models and shapley regression. Model Assisted Statistics and Applications 2:201-212

Lipovetsky S (2008) Surf - structural unduplicated reach and frequency: Latent class turf and shapley value analyses. International Journal of Information Technology and Decision Making 7:203-216

Lipovetsky S, Conklin M (2001) Analysis of regression in game theory approach. Applied Stochastic Models in Business and Industry 17:319-330

Lipovetsky S, Conklin M (2010) Reply to the paper 'do not adjust coefficients in shapley value regression'. Applied Stochastic Models in Business and Industry 26:203-204

Moulin H (1988) Axioms of cooperative decision making. Cambridge University Press

Pintér M (2011) Young's axiomatization of the shapley value - a new proof. International Journal of Game Theory Forthcoming

Powaga MCK, Lipovetsky S (2004) Customer satisfaction analysis: Identification of key drivers. European Journal of Operational Research 154:819-827

Shapley LS (1953) A value for $n$-person games. In: Kuhn HW, Tucker AW (eds) Contributions to the Theory of Games II, Annals of Mathematics Studies, vol 28, Princeton University Press, Princeton, pp 307-317

Stufken J (1992) On hierarchical partitioning. The American Statistician 46:70-71

Young HP (1985) Monotonic solutions of cooperative games. International Journal of Game Theory 14:65-72 